CFreund Publishing House Ltd International Journal of Nonlinear Sciences and Jumerical Simulation 3, 699-702, 2002

\title{
Size Effects in Micro-bending Deformation of MEMS Devices Based on the Discrete Dislocation Theory
}

\section{Shan Tang}

Lnm, Institute of Mechanics, Chinese Academy of Sciences, Beijing 100080, China, E-mail: ts_tangshan@Hotmail.com

\section{Chen Zhu}

Lnm, Institute of Mechanics, Chinese Academy of Sciences, Beijing 100080, China, E-mail: Zc@Lnm.imech.ac.cn

\section{Yue-Guang Wei}

Lnm, Institute of Mechanics, Chinese Academy of Sciences, Beijing 100080, China, E-mail: Ywei@Lnm.imech.ac.cn (Corresponding Author)

\begin{abstract}
In the present research, the discrete dislocation theory is used to analyze the size effect phenomena for the MEMS devices undergoing micro-bending load. A consistent result with the experimental one in literature is obtained. In order to check the effectiveness to use the discrete dislocation theory in predicting the size effect, both the basic version theory and the updated one are adopted simultaneously. The normalized stress-strain relations of the material are obtained for different plate thickness or for different obstacle density. The prediction results are compared with experimental results.
\end{abstract}

Keywords: Micro-bending, devices, size effects, discrete dislocation theory

\section{Introduction}

The research of MEMS device undergoing the micro-mechanical loading (such as microtorsion and micro-bend) has been becoming a key task for the device design and property assessments, and it has been attracting the great deal of interest in the research region [1]. Unlike the conventional torsion and bend of macroscopic device specimen, the torsion and bend of the MEMS device behave a high mechanical strength due to several-order smaller size for the MEMS device than that for the conventional one. Such property differences between a conventional scale device and a micro-scale device are usually referred to as the size effects. About size effect, one can not simulate it by adopting the conventional elasticplastic theory, since no any length parameters are included. In order to simulate the size effects, several theoretical methods have been presented. One kind of theories is the strain gradient theory [2]. About the theory, an advantage is that some material length parameters are included. When the theory is used to predict material behaviors in different scales, the strain gradient effect behaves considerable influences on the results. Length scale parameters characterize the strain gradient strength. Specifically, when the theory is applied to the macroscale problem, the strain gradient effect is so weak that the prediction of the strain gradient theory is consistent with that of the conventional theory. However, when the strain gradient theory is applied to the 
microscale problem, the strain gradient effect is strong, so that the size effect can be captured. Another kind of theories is the discrete dislocation theory (DD theory) [3 5]. By the theory, size effect is interpreted as the competition result of the discrete dislocation stress field with boundary effect. The boundary effect is referred to as the interaction effect from true material boundary and sub-region boundary. It is closely depended on the material size and the sub-region size as well as dislocation density. Using the $\mathrm{DD}$ theory, the micro-bending problem for beam has been researched [4]. However, the theoretical result was not consistent with the experimental observation [5].

In the present research, the DD theory is used to analyze the size effect phenomena for the MEMS device undergoing the microbending by using a different bending boundary condition from that adopted in [4]. A consistent result with the experimental one in [5] is obtained. In order to check the effectiveness to use the DD theory in predicting the size effect, in the present research we use both the basic version of the theory [6] and the updated one [3] to the micro-bending problem. The normal stress-strain relations of the material are obtained for different plate thickness or for different obstacle density. The prediction results will compare with experimental results.

\section{Fundamental Equations for DD Theory}

The discrete dislocation theory can be briefly summarized as follows. Firstly, assume that when a body is subject to some kind of loading, and inside the body region some discrete dislocations are produced (elastic deformation plus the discrete dislocation). The total mechanics fields can be divided into two parts,

$$
\mathbf{u}=\tilde{\mathbf{u}}+\hat{\mathbf{u}}, \varepsilon=\widetilde{\varepsilon}+\hat{\varepsilon}, \quad \sigma=\tilde{\sigma}+\hat{\sigma}
$$

The $(\widetilde{\mathbf{u}}, \widetilde{\boldsymbol{\varepsilon}}, \widetilde{\sigma})$ field is the field associated with the discrete dislocation in an infinitely large medium of the homogeneous matrix material.
Corresponding governing equations for the field can be described by

$$
\left.\begin{array}{ll}
\nabla \cdot \widetilde{\boldsymbol{\sigma}}=\mathbf{0} & \widetilde{\boldsymbol{\varepsilon}}=\nabla \tilde{\mathbf{u}} \\
\tilde{\boldsymbol{\sigma}}=\mathbf{L}: \widetilde{\boldsymbol{\varepsilon}} &
\end{array}\right\}
$$

Boundary conditions are dictated as

$$
\mathbf{n} \cdot \widetilde{\boldsymbol{\sigma}}=\widetilde{\mathbf{T}} \text {, on } \mathrm{S}_{\mathrm{f}} ; \mathbf{u}=\widetilde{\mathbf{U}}, \quad \text { on } \mathrm{S}_{\mathrm{u}}
$$

The $(\hat{\mathbf{u}}, \hat{\boldsymbol{\varepsilon}}, \hat{\sigma})$ field is the field to correct for the actual boundary conditions as well as for the presence of the inclusions. The problem formulations can be described as follows

$$
\begin{array}{lr}
\left.\begin{array}{l}
\boldsymbol{\nabla} \cdot \hat{\boldsymbol{\sigma}}=\mathbf{0} \\
\hat{\boldsymbol{\varepsilon}}=\nabla \hat{\mathbf{u}}
\end{array}\right\} & \text { in } \mathrm{V} \\
\hat{\boldsymbol{\sigma}}=\mathbf{L}: \hat{\boldsymbol{\varepsilon}}, & \\
\hat{\boldsymbol{\sigma}}=\mathbf{L}^{*}: \hat{\boldsymbol{\varepsilon}}+\left(\mathbf{L}^{*}-\mathbf{L}\right): \widetilde{\boldsymbol{\varepsilon}}, & \text { in } \mathrm{V}^{*} \\
\mathbf{n} \cdot \hat{\boldsymbol{\sigma}}=\mathbf{T}_{\mathbf{0}}-\widetilde{\mathbf{T}}, & \text { on } \mathrm{S}_{\mathrm{f}} \\
\mathbf{u}=\mathbf{u}_{\mathbf{0}}-\widetilde{\mathbf{U}}, & \text { on } S_{\mathrm{u}}
\end{array}
$$

Furthermore, the Peach-Koehler force acting on each dislocation determining the motion of the dislocation can be calculated.

\section{Micro-Bending Results and Analyses}

Using the DD theory to study the basic mechanical problems has had a long history [6]. In the previous researches, most of them tried to use the dislocation method result for interpreting the conventional elastic-plastic theory result, such as: work hardening, ductility, brittle, etc. In the present research, the previous DD theory version [6] will be used to study size effect for the micro-bending problem first. Then, the updated version model [3] will be used to predict the size effect phenomenon in detail.

\subsection{Application of the previous version of the DD theory to size-effect prediction}

The result for the micro-bend problem is shown in Figure 1. Where, $M$ is moment, $t$ is plate thickness, $b$ is plate width, $G$ is shear 
modulus, $\mathrm{c}$ is elastic zone size, $\sigma_{Y}$ is material yielding strength. For comparison, in Figure 1, the experimental result of thin plate bend for material $\mathrm{Ni}$ [7] is also shown. Through comparing both results, one finds that the size effect phenomenon in the micro-bend problem can be predicted successfully by adopting the discrete dislocation model.

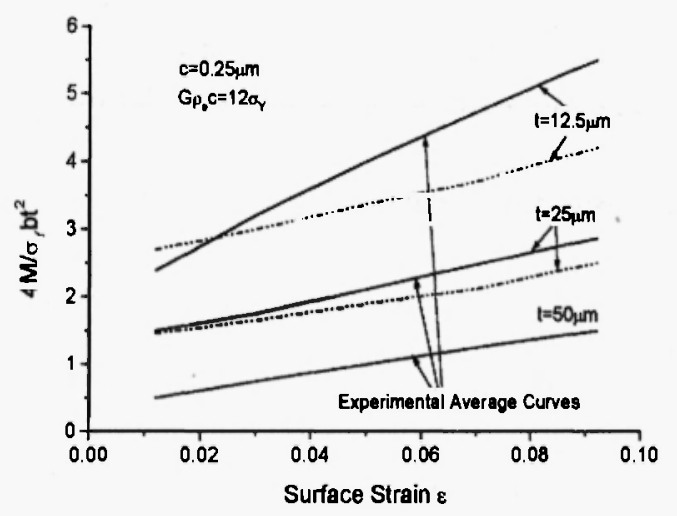

Figure 1. Comparison of the DD theory result with experimental result for $\mathrm{Ni}$ thin plate micro-bending of $\mathrm{Ni}$ specimen [7]. Dashed lines are the DD theory results.

\subsection{The DD general theory application}

The micro-bending problem is treated as the plane strain problem here. In [4], the microbending boundary condition was realized by exerting a varying displacement distribution and an additional uniform tension on the right and left terminal boundaries to keep the terminal surfaces as planes after deformation. However, the obtained result is inconsistent with the experimental measurement and observation [5], such that the dislocation can not move across the middle plane of bending. In the present research, a linear distributed traction is considered to apply to the terminal surfaces to realize the bending condition. A finite element method based on the DD theory (Eqs (1) (6)) is adopted. In our analysis, take $\mathrm{t}=4 \mu \mathrm{m}, \mathrm{L}=12 \mu \mathrm{m}$.
Considering the randomly selected dislocation source points (total 710 points), as shown in Figure 2, under loading, the dislocation will be produced at the source points, where the maximum shear stress along the inclined plane $\beta$ obtains the critical value $\tau_{n u c}$. Dislocation can move across an obstacle when the shear stress attains a value $\tau_{o b s .}$.

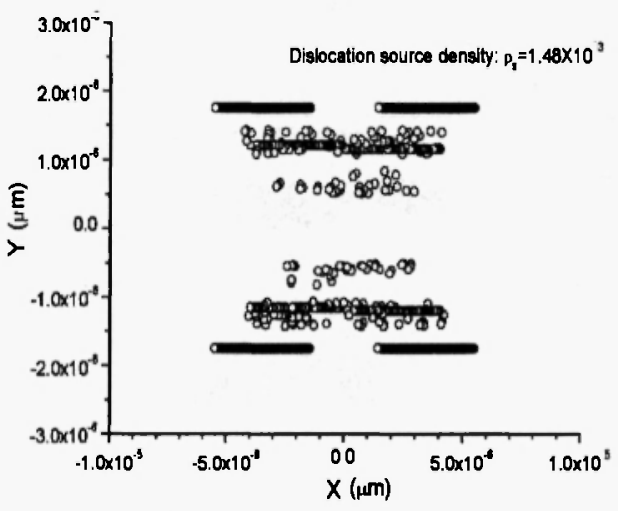

Figure 2. A randomly distributed dislocation source (total 710 points).

Figure 3 shows the dislocation distribution for three loading cases, $\mathrm{M}=1.0 \mathrm{M}_{0}, 1.5 \mathrm{M}_{0}, 1.75$ $\mathrm{M}_{0}$, where $\mathrm{M}_{0}=b t^{2} \sigma_{Y} / 6$, no obstacle case for dislocation moving is considered. Simultaneously, in our analysis, take $\tau_{n u c}=50 \mathrm{Mpa}, \quad \tau_{o b s}=150 \mathrm{Mpa}$ (obstacle). A double slip is taken into account, $\beta= \pm 30^{\circ}$.

From Figure 3, with increasing moment, dislocations are produced and moving. Especially when loading is large, the dislocation density tends to the saturation quickly. Dislocation moves without crossing the middle plane of bending. This is consistent with the experimental observation in [5].

Figure 4 shows the moment-rotation relations for three different obstacle densities. Clearly, the material property is sensitive to the obstacle density, which characterizes the material microstructure behaviors. 


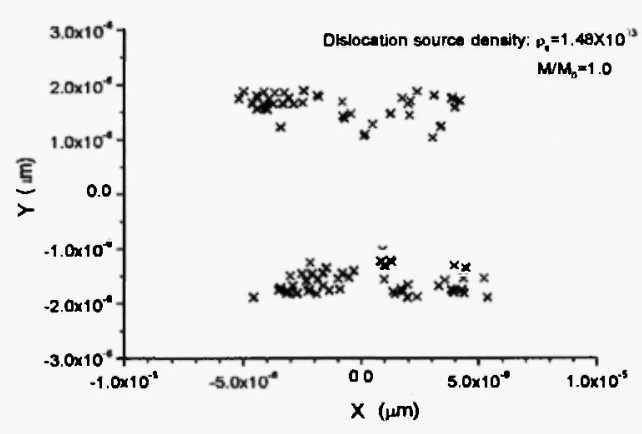

(a)

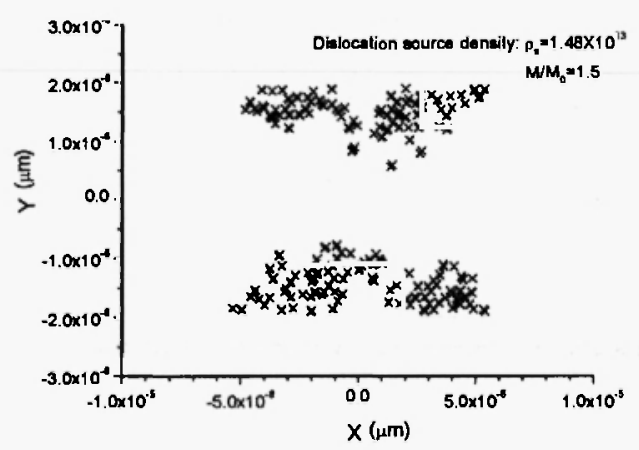

(b)

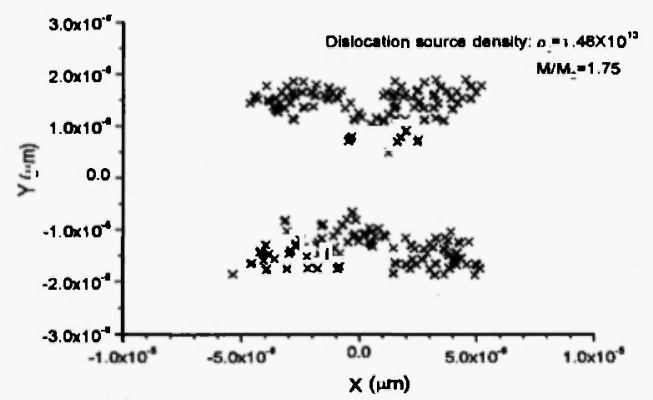

(c)

Figure 3. Dislocation distribution for reference cases, no obstacle case is considered.

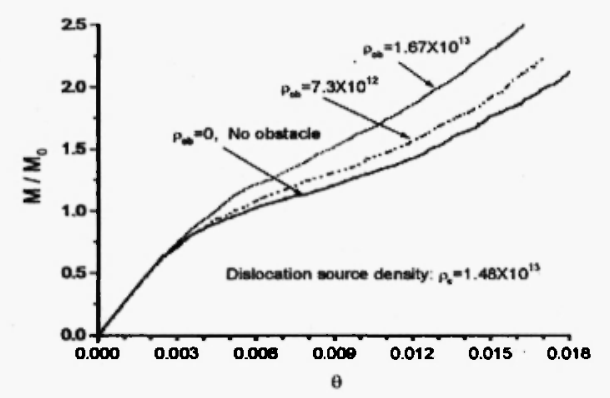

Figure 4. Moment-rotation relation for different obstacle densities.

\section{Conclusion}

Size effects in micro-bending problem of the MEMS divices have been analyzed by using the discrete dislocation theory. The present results are consistent with the experimental observation for dislocation motion law. The obstacles to resist the dislocation motion and to characterize the material microstructures influence the material behavior considerably.

\section{Acknowledgments}

This work was supported by the National Natural Science Foundation of China through Grants 19891180 and 19925211, and jointly supported by the Chinese Academy of Sciences through "Bai Ren Plan".

\section{References}

[1] Senturia, S.D. Microsystem design, Kluwer Academic Publishers, 2001

[2] Fleck, N.A., Hutchinson, J.W. Strain gradient plasticity. Advances in Applied. Mechanics, 33 (1997) 295-361

[3] Van der Giessen, E., Needleman, A. Discrete dislocation plasticity: a simple planar model, Simul Mater Sci Eng, 3 (1995)689-735

[4] Cleveringa, H.H.M., Van der Giessen, Needleman, A. A discrete dislocation analysis of bending, Int J of Plasticity, 15 (1999) 837-868

[5] Hsia, J.K. thlo International Conference of Fracture, Hawaii, USA, Dec.1-6, 2001

[6] Mura, T. Individual dislocations and continuum mechanics, In "Inelastic behavior of solids", eds. M.F. Kanninen et al., McGraw-Hill, (1970) 211230

[7] Stolken, J.S., Evans, A.G. A microbend test method for measuring the plasticity length scale, Acta Mater. 46 (1998)5109-5115 